${ }^{1}$ Clínica de la Mujer Medicina Reproductiva. Viña del Mar, Chile. 2Departamento de Pediatría, Ginecología y Medicina Preventiva, Universitat Autónoma de Barcelona. Barcelona, España.

${ }^{3}$ Unidad de Endocrinología,

Fundación Neuropsiquiátrica de Santiago (NEPSIS). Santiago,

Chile.

Los autores declaran no tener conflictos de interés.

Trabajo recibió financiamiento.

Recibido el 22 de agosto de 2019, aceptado el 3 de julio de 2020

Correspondencia a: José Moreno Sepulveda Clínica de la Mujer Medicina Reproductiva.

Alejandro Navarrete 2606, Viña del Mar. Valparaíso, Chile. jmorenos@gmail.com

\section{Dieta baja en carbohidratos y dieta cetogénica: impacto en enfermedades metabólicas y reproductivas}

\author{
JOSÉ MORENO-SEPÚLVEDA ${ }^{1,2}$, MAGDALENA CAPPONI $^{3}$
}

\section{The impact on metabolic and reproductive diseases of low-carbohydrate and ketogenic diets}

With low carbohydrate diets glucose becomes unavailable as a source of energy for our body, leading to the production of ketones from fatty acids in the liver. The increase in plasma ketones is known as nutritional ketosis. The available evidence from basic and clinical studies indicates that both low carbohydrate and high fat low carbohydrate diets are effective for weight loss and are better than non-intervention. However, low carbohydrate diet and ketogenic diets induce unique metabolic changes and consistently improve some markers of cardiovascular risk, lowering elevated blood glucose, insulin, triglycerides, ApoB and saturated fat concentrations, reducing small dense LDL particle numbers, glycated hemoglobin levels, blood pressure and body weight while increasing $H D L$-cholesterol concentrations and reversing non-alcoholic fatty liver disease. Low carbohydrate diets are an efficient strategy for the management of obesity and metabolic syndrome. They may also benefit patients with polycystic ovary syndrome. They must be prescribed by trained professionals to balance the risks and benefits for each individual patient. Future research is required to improve the knowledge about individual responses to dietary interventions, their safety, tolerance, efficacy and long-term effects.

(Rev Med Chile 2020; 148: 1630-1639)

Key words: Diet, Carbohydrate-Restricted; Diet, Ketogenic; Obesity; Metabolic Syndrome; Polycystic Ovary Syndrome.

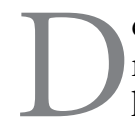
esde su divulgación en 1920, como tratamiento para la epilepsia, y más tarde para la obesidad, diversos estudios han intentado entender los mecanismos de las dietas bajas en carbohidratos (DBC) y su posible aplicación en diversas situaciones clínicas ${ }^{1-5}$. Sin embargo, también han generado controversia, puesto que mientras se reportan beneficios en diabetes mellitus tipo 2 (DM2), obesidad y síndrome metabólico; otros las consideran riesgosas y poco efectivas a largo plazo. Todo esto, durante años en los cuales existe un consenso global y guías nacionales que apoyan las dietas bajas en grasas y ricas en carbohidratos (BGRC) a fin de reducir el riesgo cardiovascular ${ }^{2}$. En los últimos años, diversas revisiones siste- máticas de la literatura han comparado los efectos de las DBC, dietas tradicionales BGRC y otras estrategias en cuanto al control del peso y los factores de riesgo cardiovascular ${ }^{4,5}$ En conjunto, estas establecen que para bajar de peso, las DBC son tan o más efectivas que las dietas BGRC ${ }^{5,6-8}$ y que además, las DBC se asocian a cambios significativos en cuanto a factores de riesgo cardiovascular ${ }^{6-8}$.

\section{Definiciones}

La dieta baja en carbohidratos (DBC) determina una inadecuada disponibilidad de glucosa como fuente energética, lo que hace necesario 
crear otra: los cuerpos cetónicos. El hígado convierte la grasa en ácidos grasos y produce los cuerpos cetónicos a partir de ésta, reemplazando a la glucosa como primera fuente energética. El aumento de cetonas es conocida como cetosis nutricional. Aunque la respuesta individual es variable, la cetosis usualmente se desencadena al restringir la ingesta de carbohidratos a menos de 50 g diarios con algún grado de restricción proteica, evitando la neoglucogénesis a partir de proteínas.

Aunque las definiciones de la DBC difieren entre sí, las que serán utilizadas en esta revisión se muestran en la Tabla 1.

Las DBC pueden ser hipocalóricas o isocalóricas según la ingesta calórica total, dependiente de factores como el contenido calórico de los carbohidratos y de la proporción de grasas y proteínas. Existen distintas pautas nutricionales que establecerán una $\mathrm{DBC}$ iso o hipercalórica, hiperproteica o alta en grasas; sin embargo, en la siguiente revisión el común denominador de las dietas estudiadas es la restricción de carbohidratos y sus variantes.

\section{Fisiología}

Los principales cuerpos cetónicos: el ácido betahidroxibutírico (bOHB), acetoacetato y acetona, se generan en el hígado por la b-oxidación de ácidos grasos libres. En condiciones habituales, la energía se origina principalmente de la glucosa proveniente de los carbohidratos. La glucosa entra en un proceso de glucólisis, generando acetil coenzima A (CoA), que se condensa con oxalacetato en el ciclo de los ácidos tricarboxílicos o ciclo de Krebs, cuyos electrones resultantes son usados en la cadena de transporte de electrones para generar fosfatos de alta energía (ATP $)^{9,10}$.

En períodos de baja ingesta de carbohidratos, como en las DBC o ayunos prolongados, se genera una falla en la glucólisis y una derivación de la CoA hacia la cetogénesis, resultando en una acumulación de cuerpos cetónicos ${ }^{9}$. El hígado expresa en abundancia HMG-CoA sintasa, una enzima clave de la cetogénesis, la cual es activada por la desacetilación mediada por Sirtuina 3 y cuyo gen HMGCS2 es regulado negativamente por la insulina a través de mecanismos transcripcionales ${ }^{11}$. Dado que la mitocondria hepática carece de enzi-
Tabla 1. Clasificación de la dieta según contribución de carbohidratos

\begin{tabular}{|lcc|}
\hline Clasificación ${ }^{\mathbf{1 3}}$ & g/día & \% ITE \\
\hline $\begin{array}{l}\text { Dieta muy baja en carbohidratos } \\
\text { o dieta cetogénica }\end{array}$ & $<50$ & $<10$ \\
Dieta baja en carbohidratos & $<130$ & $<26$ \\
\hline Dieta moderada en carbohidratos & $130-225$ & $26-45$ \\
\hline Dieta alta en carbohidratos & $>225$ & $>45$ \\
\hline
\end{tabular}

ITE: ingesta total de energía.

mas necesarias para oxidar cuerpos cetónicos, las cetonas se envían al corazón, músculo esquelético y cerebro (atraviesan la barrera hematoencefálica), quienes expresan en abundancia la enzima succinil CoA:3-cetoácido CoA-transferasa (SCOT), requerida para convertir cuerpos cetónicos a CoA, y así obtener la oxidación terminal del ciclo de Krebs ${ }^{10}$.

De ésta manera, la cetogénesis hepática ayuda a mantener la homeostasis del ciclo de krebs, previene la acumulación de ácidos grasos oxidados incompletamente, mantiene el balance redox hepático y provee a los órganos extrahepáticos de sustratos energéticos en casos de disponibilidad limitada de glucosa.

La concentración circulante de cuerpos cetónicos es determinada primeramente por la ingesta de carbohidratos y la acción de insulínica. Dentro de las diferentes DBC, en aquellas con una ingesta diaria de carbohidratos menor a $50 \mathrm{~g}$ al día se generarán mayores niveles de cetonemia. La utilización de cuerpos cetónicos como fuente de energía, a partir de la grasa, previene la necesidad de activar la neoglucogénesis hepática a partir de proteínas y de generar una disminución de la masa magra (muscular y ósea) ${ }^{10,12}$.

\section{Consideraciones iniciales}

La restricción de carbohidratos es fundamental para alcanzar cetosis nutricional. El consumo absoluto de grasas no siempre aumenta, por lo cual "dieta alta en grasas", no es un término adecuado $^{13}$.

Debido a que a mayor restricción de carbohidratos la cetosis nutricional será de mayor magnitud, se han intentado establecer indicaciones y contraindicaciones para las diferentes DBC según 
la ingesta de carbohidratos. La Tabla 2 enumeran las indicaciones de la dieta cetogénica.

A priori, las DBC no se recomiendan en pacientes con nefrolitiasis, dislipidemia y hepatopatías; pero se contraindican ante patologías del transporte de ácidos grasos, porfiria, deficiencia de piruvato-carboxilasa o usuarios de inhibidores SGLT2 $2^{12-14}$.

El aporte calórico total para cada individuo está basado en antropometría, previa ingesta dietética $\mathrm{y}$ actividad física.

Los potenciales efectos adversos que aparecen al corto plazo se agrupan en un estado popularmente conocido como "ketoflu". Estos síntomas son prevalentes durante los períodos de adaptación y tienden a desaparecer en la mayoría de los pacientes ${ }^{14,15}$.

Sin embargo, a mediano plazo pueden pro-

Tabla 2. Indicaciones para el uso de la dieta muy baja en carbohidratos o cetogénica

\begin{tabular}{|c|c|}
\hline \multicolumn{2}{|c|}{ Indicaciones para el uso de la dieta cetogénica } \\
\hline $\begin{array}{l}\text { Enfermedades } \\
\text { endocrinas }{ }^{13,36,46,70}\end{array}$ & $\begin{array}{l}\text { Diabetes mellitus tipo } 2 \\
\text { Obesidad } \\
\text { Síndrome metabólico } \\
\text { Síndrome de ovario poliquístico } \\
\text { Hiperinsulinismo congenito } \\
\text { Hígado graso no alcohólico }\end{array}$ \\
\hline $\begin{array}{l}\text { Enfermedades } \\
\text { neurológicas }{ }^{13,27}\end{array}$ & $\begin{array}{l}\text { Epilepsia refractaria } \\
\text { Epilepsia mioclónica astática } \\
\text { Enfermedad de Parkinson } \\
\text { Enfermedad de Alzheimer } \\
\text { Esclerosis lateral amiotrófica } \\
\text { Migraña } \\
\text { Narcolepsia } \\
\text { Depresión } \\
\text { Autismo }\end{array}$ \\
\hline $\begin{array}{l}\text { Enfermedades } \\
\text { metabólicas }^{13}\end{array}$ & $\begin{array}{l}\text { Deficiencia del transportador de } \\
\text { glucosa tipo } 1 \\
\text { Deficiencia del complejo piruvato } \\
\text { deshidrogenasa } \\
\text { Deficiencia de fosfofructocinasa }\end{array}$ \\
\hline $\operatorname{Otras}^{13,28}$ & $\begin{array}{l}\text { Trauma e isquemia } \\
\text { Cáncer } \\
\text { Acné }\end{array}$ \\
\hline
\end{tabular}

ducirse algunas deficiencias de minerales como potasio y magnesio, dependiendo del grado de restricción de ciertos alimentos ${ }^{14}$. Se debe prevenir la deshidratación, pues la excreción de agua y sodio aumentan por excreción urinaria de cuerpos cetónicos y disminución de la acción insulínica a nivel renal. En caso de DBC muy restrictivas se deben prescribir suplementos de calcio, selenio, zinc, vitamina $\mathrm{D}$ y citrato potásico para reducir la incidencia de deficiencias y nefrolitiasis ${ }^{16}$.

En cuanto a las dislipidemias, se ha reportado inicialmente un aumento del colesterol total y LDL en DBC con predominio de grasas saturadas, lo que sería clínicamente irrelevante ${ }^{17,18}$, puesto que a mediano plazo se observa disminución del LDL, aumento del HDL, disminución de TG y aumento del volumen y tamaño de partículas de LDL, reduciendo el riesgo cardiovascular por disminución de la aterogenicidad ${ }^{19}$. Sin embargo no se han desarrollado estudios aún sobre el impacto de las DBC en pacientes con hipercolesterolemia familiar, por tanto se recomienda tomar precauciones debido al riesgo de hipertrigliceridemia y pancreatitis aguda.

En la Tabla 3 se enumeran los efectos adversos de la cetosis nutricional.

Aunque estudios observacionales muestran que DBC y dietas BGRC tienen adherencias similares ${ }^{20}$, una reciente revisión sistemática reportó una tasa de deserción mayor en las dietas BGRC 6 .

Pese a que estudios a largo plazo en ratas reportaron una disminución en masa pancreatica y osea ${ }^{21,22}$, estudios en humanos han mostrado resultados contradictorios. Por un lado, un extenso estudio de cohortes retrospectivo en mujeres australianas de entre 50 y 67 años, reportó que la

\section{Tabla 3. Efectos adversos de la cetosis nutricional}

\begin{tabular}{|ll|}
\hline $\begin{array}{l}\text { Efectos adversos del } \\
\text { corto plazo }{ }^{\mathbf{1 4}-16}\end{array}$ & $\begin{array}{l}\text { Efectos adversos del } \\
\text { largo plazo }\end{array}$ \\
\hline Náuseas y vómitos & $\begin{array}{l}\text { Anomalías del metabolismo } \\
\text { de los lípidos }\end{array}$ \\
\hline Estreñimiento & Esteatosis hepática severa \\
\hline Deshidración & Hipoproteinemia \\
\hline Anorexia & Deficiencias minerales \\
\hline Letargo & Miocardiopatía \\
\hline Hipoglucemia & Nefrolitiasis \\
\hline
\end{tabular}


restricción de carbohidratos a largo plazo se asoció a un aumento de diabetes tipo $2^{23}$; pero por otro lado recientes publicaciones, incluyendo un ensayo clínico randomizado no han identificado efectos metabólicos deletéreos en DBC con una adherencia mayor a 6 meses $^{24-26}$.

Un error común es confundir la cetosis nutricional con la cetosis diabética. En las DBC se genera cetosis nutricional sin cambios del $\mathrm{pH}$ sanguíneo, correspondiendo a una respuesta fisiológica normal, también presente al ayunar, período neonatal y embarazo ${ }^{27}$. En cambio en diabéticos con déficit absoluto de insulina, la cetosis se establece por falta de mecanismos compensatorios e inhibitorios de cuerpos cetónicos, asociándose a acidosis. La concentración sanguínea habitual de cuerpos cetónicos es de 0,1-0,3 mmol/L. En cetosis nutricional estos niveles alcanzan entre 1-8 mmol/L. En contraste en cetoacidosis diabética se exceden los $25 \mathrm{mmol} / \mathrm{L}$ y el ph disminuye bajo 7,3 . No existe riesgo de cetoacidosis en individuos sin diabetes ${ }^{27}$.

\section{Beneficios reportados}

Los cuerpos cetónicos constituyen una super fuente de energía debido al efecto favorable sobre el metabolismo celular de distintos tejidos. Se reportan beneficios en epilepsia y obesidad; además de promisorios resultados preliminares en desórdenes neurológicos, psiquiátricos, acné y cáncer ${ }^{20,27-29}$. Además activan el gen FGF21 (por su nombre en inglés fibroblast growth factor 21), que modula la lipólisis, eleva concentraciones de vitamina $\mathrm{D}$ y disminuye $\mathrm{TG}^{12,30}$. Investigaciones en ratones muestran modificaciones de la función mitocondrial y microbiota, optimizando funciones neurovasculares y disminuyendo radicales libres y neurodegeneración ${ }^{31-33}$.

\section{Efecto de las DBC sobre patologías metabólicas}

\section{Obesidad}

Desde 1860 existen registros de prescripción de DBC como estrategia para bajar de peso, resurgiendo el interés en 1972 con la dieta Atkins, que sin embargo fue desprestigiada debido a las grasas saturadas y sus riesgos. Durante la última década han aparecido nuevas revisiones sistemáti- cas comparando DBC y dietas BGRC para bajar de peso; reportando una eficacia similar ${ }^{3,4}$, o mejores resultados con $\mathrm{DBC}^{5-8}$.

Un ensayo clínico randomizado de 148 adultos obesos sin diabetes o enfermedad cardiovascular con dieta BGRC ad libitum versus DBC, mostró que el grupo con $\mathrm{DBC}$ perdió significativamente más peso a los 12 meses $(5,3 \mathrm{~kg} \text { versus } 1,8 \mathrm{~kg})^{34}$. Resultados similares a favor de DBC se han reportado en adolescentes ${ }^{17}$, adultos diabéticos ${ }^{35,36}$ y adultos sin diabetes ${ }^{37,38}$, y adultos sin restricción calórica $^{39}$, todos evaluados en ensayos clínicos randomizados con seguimiento de 6 meses a 2 años. En esta línea, un reciente metaanálisis de ensayos clínicos randomizados sugiere que la disminución de peso es más marcada a mediano y largo plazo (hasta 24 meses) en DBC versus dietas BGRC ${ }^{8}$.

En la Tabla 4 se muestra el efecto de la dieta cetogénica sobre algunos factores de riesgo cardiovascular en pacientes con sobrepeso u obesidad al año de seguimiento según el metaanálisis de Bueno et $\mathrm{al}^{5}$.

La adherencia es un tópico fundamental en las $\mathrm{DBC}$, pues se ha observado una subsecuente alza de peso si se aumentan los carbohidratos ingeridos (rebote), secundaria a la descontinuación de la cetosis ${ }^{13}$. Monitorizando estrictamente este indicador, obtenemos bajas de peso de $12 \%$ en DBC versus 3-7\% en dietas $\mathrm{BGRC}^{40}$. Un estudio reportó pérdidas de hasta $80 \mathrm{~kg}$ en $\mathrm{DBC}^{41}$.

Dos mecanismos explicarían estos resultados. Por un lado, existiría un incremento de la saciedad determinado por la cetosis, generando modulación de la leptina y adiponectina (hormonas

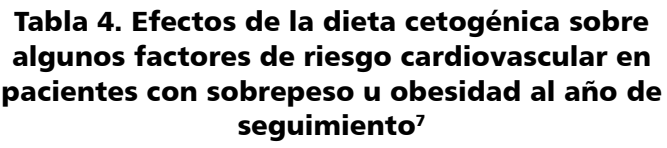

\begin{tabular}{|llc|}
\hline Marcador & Efecto & $\begin{array}{c}\text { Diferencia promedio } \\
\text { (IC 95\%) }\end{array}$ \\
\hline Peso $(\mathrm{kg})$ & Disminuye & $-0,91(-1,65 ;-0,17)$ \\
\hline TG $(\mathrm{mmol} / \mathrm{l})$ & Disminuye & $-0,18(-0,27 ;-0,08)$ \\
\hline HDL $(\mathrm{mmol} / \mathrm{l})$ & Aumenta & $0,09(0,06 ; 0,12)$ \\
LDL $(\mathrm{mmol} / \mathrm{l})$ & Aumenta & $0,12(0,04 ; 0,20)$ \\
PAS $(\mathrm{mmHg})$ & No significativo & $-1,47(-3,44 ; 0,50)$ \\
\hline PAD $(\mathrm{mmHg})$ & Disminuye & $-1,43(-2,43 ;-0,37)$ \\
\hline
\end{tabular}

PAS: presión arterial sistolica; PAD: presión arterial diastólica. 
reguladoras del apetito), y menores instancias de hipoglucemia, causa común de hambre dietas BGRC, especialmente si padecen de insulinorresistencia (IR $)^{31,42}$. Por otra parte, las DBC determinarían un giro de proteínas a gluconeogénesis y aumento de lipólisis con reducción de lipogénesis, constituyendo una ventaja metabólica. Este efecto sería dependiente de si la insulinemia está disminuida, lo cual se consigue con $\mathrm{DBC}^{43}$.

En la Tabla 5 se resumen los diferentes mecanismos que explicarían los cambios metabólicos y ponderales asociados a la cetosis nutricional.

\section{Diabetes Mellitus Tipo 2}

Previo al descubrimiento de la insulina, la restricción de carbohidratos asociada con ayuno fue el plan alimentario prescrito en DM2 ${ }^{44,45}$. Hoy, las DBC están siendo incluidas nuevamente como tratamiento de primera línea ${ }^{46,47}$. Un estudio de 363 pacientes obesos y sobrepeso, comparó baja de peso, control glicemico y HbAlc tras prescribir DBC versus dieta hipocalórica. Tanto en diabéticos como no diabéticos hubo mayor baja de peso con DBC; además, en los diabéticos el control glicémico y $\mathrm{HbAlc}$ fueron significativamente mejores con $\mathrm{DBC}^{48}$.

Otros estudios han reportado mejoras significativas en la glicemia en ayunas, la insulinemia en ayunas y los niveles de péptido $\mathrm{C}$ durante los primeros días de las DBC hipocalóricas ${ }^{49,50}$.

Un estudio randomizó a 34 pacientes DM2 a dieta hipocalórica versus dieta cetogénica con ingesta calórica ad libitum. El grupo con dieta cetogénica presentó a los 3 meses una mayor disminución de $\mathrm{HbAlc}$ (6,0\% vs 6,9\%), mayor pérdida de peso $(-5,5 \mathrm{vs}-2,6 \mathrm{~kg})$ y una disminución significativa del uso de medicamentos hipoglicemiantes ${ }^{51}$. Resultados similares se observaron en el estudio de Westman et al. que comparó dieta cetogénica versus una dieta baja en índice glicémico. El grupo con dieta cetogénica experimentó un descenso significativamente mayor en $\mathrm{HbAlc}(-1,5 \%$ vs $-0,5 \%)$ y una reducción mayor de medicamentos hipoglicemiantes ( $95 \%$ vs $62 \%$ ) a las 24 semanas ${ }^{15}$.

Un reciente estudio prospectivo de un año de seguimiento, reportó que pacientes diabéticos tipo 2 obtienen mejores resultados en cuanto a reducción de $\operatorname{HbAlc}(-1,29 \%)$, disminución ponderal $(-12,8 \mathrm{~kg})$ al comparar cetosis nutricional versus cuidados habituales ${ }^{52}$. Además $100 \%$ de los pacientes insulinorequirientes con cetosis
Tabla 5. Mecanismos de la cetosis nutricional que generan disminución de peso y modulación metabólica

Aumento del gasto energético ${ }^{12,30}$
Aumento de la actividad simpática por acción del FGF21
Termogénesis postprandial y del metabolismo en reposo
Reducción del apetito ${ }^{31,42}$
Supresión del apetito inducida por ingesta proteica
Reducción del grelina
Aumento del peptido YY
Por acción del bOHB
Cambios en el metabolismo lipídico ${ }^{43}$
Reducción en la lipogénesis
Incremento de la lipolisis
Mayor eficiencia metabólica en el consumo de grasas
Efecto pancreático
Reducción en la secreción de insulina y glucagón
Mejoría del ánimo ${ }^{13,31,42}$
Aumento de la fuerza de voluntad
Aumento de la adherencia

nutricional suspendieron o redujeron dosis de hipoglucemiantes en comparación con $23 \%$ de pacientes con cuidados habituales.

Los factores que podrían influir en obtener resultados favorables son que la DBC además sea hipocalórica, menor edad de los pacientes y una menor duración de la diabetes ${ }^{49,53,54}$.

A largo plazo, estudios revelan que DBC hipocalóricas se asocian a niveles bajos de glicemia en ayunas y $\mathrm{HbAlc}$, incluso después de 18 meses de instaurada la intervención ${ }^{54}$.

En la Tabla 6 se resumen los efectos de la DBC sobre algunos factores de riesgo cardiovascular en pacientes diabéticos al año de seguimiento ${ }^{35}$.

La disminución ponderal mejora el control glicémico con cualquier dieta, pudiendo asumirse que los beneficios de DBC irán asociados a una baja de peso concomitante; sin embargo se ha observado que la restricción de carbohidratos influye positivamente en el control glicémico incluso en ausencia de baja de peso $^{55}$.

\section{Síndrome metabólico y riesgo cardiovascular}

El síndrome metabólico incluye hiperglicemia, hiperinsulinemia, IR; y se relaciona con dislipidemia aterogénica y enfermedades 
Tabla 6. Efectos de la dieta baja en carbohidratos sobre algunos factores de riesgo cardiovascular en pacientes diabéticos al año de seguimiento ${ }^{83}$

\begin{tabular}{|llc|}
\hline Marcador & Efecto & $\begin{array}{c}\text { Diferencia promedio } \\
\text { (IC 95\%) }\end{array}$ \\
\hline HbA1c $(\%)$ & Disminuye & $-0,28(-0,53 ;-0,02)$ \\
\hline Peso $(\mathrm{kg})$ & No significativo & $0,28(-1,37 ; 1,92)$ \\
\hline TG $(\mathrm{mmol} / \mathrm{l})$ & Disminuye & $-0,24(-0,35 ;-0,13)$ \\
\hline $\mathrm{HDL}(\mathrm{mmol} / \mathrm{l})$ & Aumenta & $0,06(0,04 ; 0,09)$ \\
\hline $\mathrm{LDL}(\mathrm{mmol} / \mathrm{l})$ & No significativo & $0,05(-0,10 ; 0,19)$ \\
\hline PAS $(\mathrm{mmHg})$ & Disminuye & $-2,74(-5,27 ;-0,20)$ \\
\hline PAD $(\mathrm{mmHg})$ & No significativo & $-0,99(-2,24 ; 0,25)$ \\
\hline
\end{tabular}

PAS: presion arterial sistolica; PAD: presión arterial diastólica.

cardiovasculares. Dietas ricas en carbohidratos promoverían esta condición, al favorecer el ensamblaje y procesamiento de lipoproteínas ${ }^{56,57}$. La cetosis nutricional tendría efectos beneficiosos, asociándose a un perfil lipídico no aterogénico y disminución de IR, sin observarse efectos adversos renales o hepáticos ${ }^{58,59}$.

Una preocupación común es que al aumentar la ingesta grasa en DBC, aumentaría el riesgo de enfermedades cardiovasculares; convicción originada por los resultados del estudio de Ancel Keys $^{60}$ y el consiguiente desarrollo de dietas BGRC preventivas de enfermedades cardiovasculares ${ }^{61}$. Sin embargo, reciente evidencia desestima esta teoría $^{62-64}$, sin encontrar asociación entre grasas saturadas y mortalidad o progresión de arteriosclerosis $^{65}$. De forma dispar, algunos estudios encontraron disminución o cambios no significativos en LDL $^{66}$ y otros reportaron aumento ${ }^{67}$; sin embargo, un metaanálisis reciente no mostró efectos significativos sobre $\mathrm{LDL}^{6}$. Se debe considerar además, que partículas pequeñas y densas de LDL tienen efectos más aterogénicos versus partículas grandes de LDL ${ }^{67}$; y que las concentraciones de TG se relacionan inversamente con las partículas densas de $\mathrm{LDL}^{68}$. La DBC al reducir los TG, determina una distribución menos aterogénica, con menor proporción de partículas pequeñas y densas y mayor de las grandes ${ }^{69}$.

Por otro lado, estudios encontraron una débil asociación entre ingesta de carnes rojas ultra procesadas y un aumento del riesgo de cáncer de colon $^{70}$; sin embargo, no existe evidencia de que la ingesta moderada tenga efectos cardiovasculares negativos $^{71}$.

Numerosos ensayos clínicos muestran cambios favorables en el riesgo cardiovascular al comparar DBC con dietas BGRC. Uno de ellos reportó una disminución mayor de TG (-0,58 vs $-0,22 \mathrm{mmol} / \mathrm{L})$ $\mathrm{y}$ un incremento mayor de HDL ( $+0,30$ vs $+0,07$ $\mathrm{mmol} / \mathrm{L}$ ) en $\mathrm{DBC}^{72}$. La ApoB, un marcador de riesgo para enfermedad coronaria, también disminuye más con DBC.

De todas las dietas, ninguna incrementa más el HDL como la DBC, superando a dietas con bajo índice glicémico, dietas BGRCy en algunos casos a la dieta mediterránea ${ }^{33,37,73}$.

Una dieta cetogénica a largo plazo incrementa el flujo sanguíneo mediado por dilatación arteriolar $^{74}$, disminución de biomarcadores inflamatorios ${ }^{75}$, incremento del control glucémico y reducción de $\mathrm{HbA1c}$, glicemia, insulinemia ${ }^{6}$ y disminución de grasa abdominal visceral. Estos cambios impactan positivamente el riesgo cardiovascular ${ }^{48}$.

Otro efecto único de DBC es la reversión del hígado graso no alcohólico (HGNA), condición causada por exceso de carbohidratos, especialmente fructosa, determinando una elevación de TG y VLDL, baja de HDL e IR con una inadecuada supresión de la glucosa hepática por la insulina. Pacientes con HGNA e IR, presentan daño endotelial y enfermedad aterogénica. Se ha observado una reducción de $58 \%$ a $19 \%$ en la prevalencia de síndrome metabólico en pacientes obesos tratados con DBC hasta 8 meses, indicando cuán efectiva es esta estrategia en $\mathrm{IR}^{76}$.

\section{Síndrome ovario poliquístico}

El Síndrome Ovario Poliquístico (SOP) que afecta mujeres en edad fértil, está asociado a obesidad, IR, hiperandrogenismo e infertilidad ${ }^{77}$. En pacientes con sobrepeso u obesidad el tratamiento de primera línea es modificar hábitos dietarios y actividad física ${ }^{78}$. Un estudio de 18.555 pacientes reportó que en mujeres con una alta ingesta de carbohidratos el riesgo de infertilidad con factor ovulatorio aumenta en $78 \%{ }^{79}$. Reportes recientes plantean la opción de indicar una $\mathrm{DBC}$ en pacientes SOP. Si bien existe consenso que tanto DBC como dietas BGRC se asocian a descenso ponderal, se destacan los beneficios adicionales que aportarían las DBC a este grupo de pacientes ${ }^{80,81}$. Por un lado, la restricción de carbohidratos reduce la 
insulinemia de ayuno y mejora la sensibilidad a la insulina; y por otro se disminuye el hiperandrogenismo, gracias al aumento de proteínas transportadoras de hormonas sexuales, mejorando el porcentaje de testosterona libre y la relación LH/ $\mathrm{FSH}^{82}$. Ésto determina una mejora de la función menstrual y fertilidad.

No obstante, no queda claro si DBC es efectiva sobre los resultados reproductivos, y futuros estudios clínicos randomizados se encuentran en curso para responder esta interrogante.

\section{Conclusiones}

A partir de la evidencia científica disponible, podemos concluir que tanto DBC como dietas BGRC son efectivas para bajar de peso, y son mejores que la no intervención. Además, DBC inducen cambios metabólicos únicos, incluyendo mejoras del control glucémico y de concentraciones plasmáticas de TG, HDL, ApoB y partículas aterogénicas de LDL. Por todo esto puede establecerse que $\mathrm{DBC}$ es una estrategia eficiente para el manejo de la obesidad y síndrome metabólico, además del subgrupo de pacientes SOP; sin embargo deben ser prescritas por profesionales capacitados, quienes deben evaluar los riesgos y beneficios para cada paciente en particular.

Finalmente, se requieren futuros estudios para entender de mejor forma la compleja respuesta individual a las intervenciones dietarias, seguridad, tolerancia, eficacia y efectos a largo plazo.

\section{Referencias}

1. Atkins RC. Dr Atkins diet revolution. 1st edn. New York: David McKay Company, 1972: 1-310.

2. Feinman RD, Pogozelski WK, Astrup A, Bernstein RK, Fine EJ, Westman EC, Accurso A, Frassetto L, Gower BA, McFarlane SI, et al. Dietary carbohydrate restriction as the first approach in diabetes management: critical review and evidence base. Nutrition 2015; 31: 1-13.

3. Naude CE, Schoonees A, Senekal M, Young T, Garner $\mathrm{P}$, Volmink J. Low carbohydrate versus isoenergetic balanced diets for reducing weight and cardiovascular risk: a systematic review and meta-analysis. PLoS ONE 2014; 9: e100652.

4. Johnston BC, Kanters S, Bandayrel K, Wu P, Naji F, Siemieniuk RA, et al. Comparison of weight loss among named diet programs in overweight and obese adults: a meta-analysis. JAMA 2014; 312: 923-33.

5. Bueno NB, de Melo IS, de Oliveira SL, da Rocha Ataide T. Very-low-carbohydrate ketogenic diet v. low-fat diet for long-term weight loss: a meta-analysis of randomised controlled trials. Br J Nutr 2013; 110: 1178-87.

6. Santos FL, Esteves SS, da Costa Pereira A, Yancy WS Jr, Nunes JP. Systematic review and meta-analysis of clinical trials of the effects of low carbohydrate diets on cardiovascular risk factors. Obes Rev 2012; 13: 1048-66.

7. Tobias DK, Chen M, Manson JE, Ludwig DS, Willett W, Hu FB. Effect of low-fat diet interventions versus other diet interventions on long-term weight change in adults: a systematic review and meta-analysis. Lancet Diabetes Endocrinol 2015; 3: 968-79.

8. Sackner-Bernstein J, Kanter D, Kaul S. Dietary intervention for overweight and obese adults: comparison of low-carbohydrate and low-fat diets. A meta-analysis. PLoS ONE 2015; 10: e0139817.

9. Laffel L. Ketone bodies: A review of physiology, pathophysiology and application of monitoring to diabetes. Diabetes Metab Res Rev 1999; 15: 412-26.

10. Schugar RC. Low-carbohydrate ketogenic diets, glucose homeostasis, and nonalcoholic fatty liver disease. Curr Opin Clin Nutr Metab Care 2012; 15: 374-80.

11. Shimazu T, Hirschey MD, Hua L, Dittenhafer-Reed KE, Schwer B, Lombard DB, et al. SIRT3 deacetylates mitochondrial 3-hydroxy-3-methylglutaryl CoA synthase 2 and regulates ketone body production. Cell Metab 2010; 12: 654-61.

12. Gupta L, Khandelwal D, Kalra S, Gupta P, Dutta D, Aggarwal S. Ketogenic diet in endocrine disorders: Current perspectives. J Postgrad Med 2017; 63: 242-51.

13. Noakes TD, Windt J. Evidence that supports the prescription of low-carbohydrate high-fat diets: a narrative review. Br J Sports Med 2017; 51 (2): 133-9.

14. Kossoff EH, Zupec-Kania BA, Amark PE, Ballaban-Gil KR, Christina Bergqvist AG, Blackford R, et al. Optimal clinical management of children receiving the ketogenic diet: Recommendations of the International Ketogenic Diet Study Group. Epilepsia 2009; 50: 304-17.

15. Westman EC, Yancy WS, Jr, Mavropoulos JC, Marquart M, McDuffie JR. The effect of a low-carbohydrate, ketogenic diet versus a low-glycemic index diet on glycemic control in type 2 diabetes mellitus. Nutr Metab (Lond) 2008; 5: 36.

16. McNally MA, Pyzik PL, Rubenstein JE. Empiric use of potassium citrate reduces kidney-stone incidence with the ketogenic diet. Pediatrics 2009; 124: e300-4.

17. Douris N, Melman T, Pecherer JM, Pissios P, Flier JS, Cantley LC, et al. Adaptative changes in amino acid me- 
tabolism permit normal longevity in mice consuming a low-carbohydrate ketogenic diet. Biochim Biophys. Acta 2015; 1852: 2056-65.

18. Foster GD, Wyatt HR, Hill JO, Makris AP, Rosenbaum $\mathrm{DL}$, Brill C, et al. Weight and metabolic outcomes after 2 years on a low-carbohydrate versus low-fat diet: a randomized trial. Ann Intern Med 2010; 153 (3): 147-57.

19. Wood RJ, Volek JS, Liu Y, Shachter NS, Contois JH, Fernandez ML. Carbohydrate restriction alters lipoprotein metabolism by modifying VLDL, LDL, and HDL subfraction distribution and size in overweight men. J Nutr 2006; 136: 384-9.

20. Partsalaki I, Karvela A, Spiliotis BE. Metabolic impact of a ketogenic diet compared to a hypocaloric diet in obese children and adolescents. J Pediatr Endocrinol Metab 2012; 25: 697-704.

21. Ellenbroek JH, van Dijck L, Töns HA, Rabelink TJ, Carlotti F, Ballieux BE, et al. Long-term ketogenic diet causes glucose intolerance and reduced $\beta$ - and $\alpha$-cell mass but no weight loss in mice. Am J Physiol Endocrinol Metab 2014; 306: E552-8.

22. Kosinski C, Jornayvaz FR. Effects of Ketogenic Diets on Cardiovascular Risk Factors: Evidence from Animal and Human Studies. Nutrients 2017; 19; 9. pii: E517.

23. Rayner J, D’Arcy E, Ross LJ, Hodge A, Schoenaker DAJM. Carbohydrate restriction in midlife is associated with higher risk of type 2 diabetes among Australian women: A cohort study. Nutr Metab Cardiovasc Dis 2020; 30: 400-9.

24. Mardinoglu A, Wu H, Bjornson E, Zhang C, Hakkarainen A, Räsänen SM, et al. An Integrated Understanding of the Rapid Metabolic Benefits of a Carbohydrate-Restricted Diet on Hepatic Steatosis in Humans. Cell Metab 2018; 27: 559-71.e5.

25. Luukkonen PK, Dufour S, Lyu K, Zhang XM, Hakkarainen A, Lehtimäki TE, et al. Effect of a ketogenic diet on hepatic steatosis and hepatic mitochondrial metabolism in nonalcoholic fatty liver disease. Proc Natl Acad Sci USA 2020; 117: 7347-54.

26. Brinkworth GD, Wycherley TP, Noakes M, Buckley JD, Clifton PM. Long-term effects of a very-low-carbohydrate weight-loss diet and an isocaloric low-fat diet on bone health in obese adults. Nutrition 2016; 32: 1033-6.

27. Paoli A, Rubini A, Volek JS, Grimaldi KA. Beyond weight loss: a review of the therapeutic uses of very-low-carbohydrate (ketogenic) diets. Eur J Clin Nutr 2013; 67: 789-96.

28. Klement RJ. Beneficial effects of ketogenic diets for cancer patients: a realist review with focus on evidence and confirmation. Med Oncol 2017; 34 (8): 132.

29. Murray AJ, Knight NS, Cole MA, Cochlin LE, Carter
E, Tchabanenko K, et al. Novel ketone diet enhances physical and cognitive performance. FASEB J 2016; 30: 4021-32.

30. Woo YC, Xu A, Wang Y, Lam KS. Fibroblast growth factor 21 as an emerging metabolic regulator: Clinical perspectives. Clin Endocrinol (Oxf) 2013; 78: 489-96.

31. Maalouf M, Rho JM, Mattson MP. The neuroprotective properties of calorie restriction, the ketogenic diet, and ketone bodies. Brain Res Rev 2009; 59 (2): 293-315.

32. Ma D, Wang AC, Parikh I, Green SJ, Hoffman JD, Chlipala G, et al. Ketogenic diet enhances neurovascular function with altered gut microbiome in young healthy mice. Sci Rep 2018; 8 (1): 6670.

33. Newman JC, Covarrubias AJ, Zhao M, Yu X, Gut P, $\mathrm{Ng} \mathrm{CP}$, et al. Ketogenic Diet Reduces Midlife Mortality and Improves Memory in Aging Mice. Cell Metabolism 2017; 26: 547-57.

34. Bazzano LA, Hu T, Reynolds K, Yao L, Bunol C, Liu Y, et al. Effects of low-carbohydrate and low-fat diets: a randomized trial. Ann Intern Med 2014; 161: 309-18.

35. Saslow LR, Kim S, Daubenmier JJ, Moskowitz JT, Phinney SD, Goldman V, et al. A randomized pilot trial of a moderate carbohydrate diet compared to a very low carbohydrate diet in overweight or obese individuals with type 2 diabetes mellitus or prediabetes. PLoS ONE 2014; 9: e91027.

36. Huntriss R, Campbell M, Bedwell C. The interpretation and effect of a low-carbohydrate diet in the management of type 2 diabetes: a systematic review and meta-analysis of randomised controlled trials. Eur J Clin Nutr 2018; 72 (3): 311-25.

37. Foster GD, Wyatt HR, Hill JO, McGuckin BG, Brill C, Mohammed BS, Szapary PO, Rader DJ, Edman JS, Klein S. A randomized trial of a low-carbohydrate diet for obesity. N Engl J Med 2003;348:2082-90.

38. Yancy WS Jr, Olsen MK, Guyton JR, Bakst RP, Westman EC. A low-carbohydrate, ketogenic diet versus a low-fat diet to treat obesity and hyperlipidemia: a randomized, controlled trial. Ann Intern Med 2004; 140: 769-77.

39. Gardner CD, Kiazand A, Alhassan S, Kim S, Stafford RS, Balise RR, et al. Comparison of the Atkins, Zone, Ornish, and LEARN diets for change in weight and related risk factors among overweight premenopausal women: the A to Z Weight Loss Study: a randomized trial. JAMA 2007; 297: 969-77.

40. Noakes TD. Low-carbohydrate and high-fat intake can manage obesity and associated conditions: occasional survey. S Afr Med J 2013; 103: 826-30.

41. Weigle DS, Breen PA, Matthys CC, Callahan HS, Meeuws KE, Burden VR, et al. A high-protein diet induces sustained reductions in appetite, ad libitum caloric 
intake, and body weight despite compensatory changes in diurnal plasma leptin and ghrelin concentrations. Am J Clin Nutr 2005; 82: 41-8.

42. Gibson A, Seimon RV, Lee CM, Ayre J, Franklin J, Markovic TP, et al. Do ketogenic diets really suppress appetite? A systematic review and meta-analysis. Obes Rev 2015; 16 (1): 64-76.

43. Volek JS, Freidenreich DJ, Saenz C, Kunces LJ, Creighton BC, Bartley JM, et al. Metabolic characteristics of keto-adapted ultra-endurance runners. Metab Clin Exp 2016; 65: 100-10.

44. Cersosimo E, Triplitt C, Solis-Herrera C, Mandarino LJ, DeFronzo RA. Pathogenesis of type 2 diabetes mellitus. In: De Groot LJ, Beck-Peccoz P, Chrousos G, et al, eds. Endotext. S Dartmouth, MA: MDText.com, 2000.

45. Allen FM, Stillman E, Fitz R. Total dietary regulation in the treatment of diabetes. No. 11. New York: The Rockerfeller Institute for Medical Research 1919; 1-650.

46. Schofield G, Henderson G, Thornley S. Very low-carbohydrate diets in the management of diabetes revisited. NZ Med J 2016; 129: 67-73.

47. Tay J, Thompson CH, Luscombe-Marsh ND, Wycherley TP, Noakes M, Buckley JD, et al. Effects of energyrestricted low-carbohydrate, high unsaturated fat/low saturated fat diet versus a high-carbohydrate, low-fat diet in type 2 diabetes: a 2-year randomized clinical trial. Diabetes Obes Metab 2018; 20: 858-71.

48. Hussain TA, Mathew TC, Dashti AA, Asfar S, Al-Zaid N, Dashti HM. Effect of low-calorie versus low-carbohydrate ketogenic diet in type 2 diabetes. Nutrition 2012; 28: 1016-21.

49. Lim EL, Hollingsworth KG, Aribisala BS, Chen MJ, Mathers JC, Taylor R. Reversal of type 2 diabetes: normalisation of beta cell function in association with decreased pancreas and liver triacylglycerol. Diabetologia 2011; 54: 2506-14.

50. Malandrucco I, Pasqualetti P, Giordani I, Manfellotto D, De Marco F, Alegiani F, et al. Very-low-calorie diet: a quick therapeutic tool to improve $\beta$ cell function in morbidly obese patients with type 2 diabetes. Am J Clin Nutr 2012; 95: 609-13.

51. Daly ME, Paisey R, Paisey R. Short-term effects of severe dietary carbohydrate-restriction advice in Type 2 diabetes-a randomized controlled trial. Diabet Med 2006; 23 : 15-20.

52. Ahmed SR, Bellamkonda S, Zilbermint M, Wang J, Kalyani RR. Effects of the low carbohydrate, high fat diet on glycemic control and body weight in patients with type 2 diabetes: experience from a community-based cohort. BMJ Open Diabetes Res Care 2020; 8. pii: e000980.

53. Rothberg AE, McEwen LN, Kraftson AT, Fowler CE,
Herman WH. Very-low-energy diet for type 2 diabetes: an underutilized therapy? J Diabetes Complications 2014; 28: 506-10.

54. Jazet IM, de Craen AJ, van Schie EM, Meinders AE. Sustained beneficial metabolic effects 18 months after a 30-day very low calorie diet in severely obese, insulin-treated patients with type 2 diabetes. Diabetes Res Clin Pract 2007;77:70-6.

55. Gannon MC, Nuttall FQ. Control of blood glucose in type 2 diabetes without weight loss by modification of diet composition. Nutr Metab (Lond) 2006; 3: 16.

56. Malik VS, Schulze MB, Hu FB. Intake of sugar-sweetened beverages and weight gain: a systematic review. Am J Clin Nutr 2006; 84: 274-88.

57. Johnson RJ, Segal MS, Sautin Y, Nakagawa T, Feig DI, Kang DH, et al. Potential role of sugar (fructose) in the epidemic of hypertension, obesity and the metabolic syndrome, diabetes, kidney disease, and cardiovascular disease. Am J Clin Nutr 2007; 86: 899-906.

58. Sartorius K, Sartorius B, Madiba TE, Stefan C. Does high-carbohydrate intake lead to increased risk of obesity? A systematic review and meta-analysis. BMJ Open 2018;8:e018449.

59. Kemper MF, Srivastava S, Todd King M, Clarke K, Veech RL, Pawlosky RJ. An ester of $\beta$-hydroxybutyrate regulates cholesterol biosynthesis in rats and a cholesterol biomarker in humans. Lipids 2015; 50: 1185-93.

60. Keys A, Menotti A, Karvonen MJ, Aravanis C, Blackburn $\mathrm{H}$, Buzina R, et al. The diet and 15-year death rate in the seven countries study. Am J Epidemiol 1986; 124: 90315.

61. Harcombe Z, Baker JS, Cooper SM, Davies B, Sculthorpe N, DiNicolantonio JJ, Grace F, et al. Evidence from randomised controlled trials did not support the introduction of dietary fat guidelines in 1977 and 1983: a systematic review and meta-analysis. Open Heart 2015; 2: e000196.

62. de Souza RJ, Mente A, Maroleanu A, Cozma AI, Ha V, Kishibe $\mathrm{T}$, et al. Intake of saturated and trans unsaturated fatty acids and risk of all cause mortality, cardiovascular disease, and type 2 diabetes: systematic review and meta-analysis of observational studies. BMJ 2015; 351: h3978.

63. Praagman J, Beulens JW, Alssema M, Zock PL, Wanders AJ, Sluijs I, et al. The association between dietary saturated fatty acids and ischemic heart disease depends on the type and source of fatty acid in the European Prospective Investigation into Cancer and Nutrition-Netherlands cohort. Am J Clin Nutr 2016; 103: 356-65.

64. Puaschitz NG, Strand E, Norekvål TM, Dierkes J, Dahl L, Svingen GF, et al. Dietary intake of saturated fat is not 
associated with risk of coronary events or mortality in patients with established coronary artery disease. J Nutr 2015; 145: 299-305.

65. Forsythe CE, Phinney SD, Feinman RD, Volk BM, Freidenreich D, Quann E, et al. Limited effect of dietary saturated fat on plasma saturated fat in the context of a low carbohydrate diet. Lipids 2010; 45: 47-62.

66. Hernández TL, Sutherland JP, Wolfe P, Allian-Sauer M, Capell WH, Talley ND, et al. Lack of suppression of circulating free fatty acids and hypercholesterolemia during weight loss on a high-fat, low-carbohydrate diet. Am J Clin Nutr 2010; 91: 578-85.

67. Superko RH. Lipoprotein subclasses and atherosclerosis. Front Biosci 2001; 6: D355-65.

68. Boizel R, Benhamou PY, Lardy B, Laporte F, Foulon T, Halimi S. Ratio of triglycerides to HDL cholesterol is an indicator of LDL particle size in patients with type 2 diabetes and normal HDL cholesterol levels. Diabetes Care 2000; 23: 1679-85.

69. Sniderman AD, Blank D, Zakarian R, Bergeron J, Frohlich J. Triglycerides and small dense LDL: the twin Achilles heels of the Friedewald formula. Clin Biochem 2003; 36: 499-504.

70. Neuschwander-Tetri BA. Carbohydrate intake and nonalcoholic fatty liver disease. Curr Opin Clin Nutr Metab Care 2013; 16: 446-52.

71. Aykan NF. Red meat and colorectal cancer. Oncol Rev 2015;9:288.

72. O'Connor LE, Kim JE, Campbell WW. Consuming $<$ or $>0.5$ servings of red meat per day does not have a negative impact on cardiovascular disease risk factors; a systematic review and meta-analysis of randomized controlled trials. FASEB J 2016; 30: S904.1.

73. Tay J, Brinkworth GD, Noakes M, Keogh J, Clifton PM. Metabolic effects of weight loss on a very-low-carbohydrate diet compared with an isocaloric high-carbohydrate diet in abdominally obese subjects. J Am Coll Cardiol 2008; 51: 59-67.
74. Feinman RD, Volek JS, Westman EC. Dietary carbohydrate restriction in the treatment of diabetes and metabolic syndrome. Clin Nutr Insight 2008; 34: 5.

75. Forsythe CE, Phinney SD, Fernández ML, Quann EE, Wood RJ, Bibus DM, et al. Comparison of low fat and low carbohydrate diets on circulating fatty acid composition and markers of inflammation. Lipids 2008; 43: 65-77.

76. Yki-Jarvinen H. Non-alcoholic fatty liver disease as a cause and a consequence of metabolic syndrome. Lancet Diabetes Endocrinol 2014; 2: 901-10.

77. McCartney CR, Marshall JC. Clinical practice. Polycystic ovary syndrome. N Engl J Med 2016; 375: 54-64.

78. Altieri P, Cavazza C, Pasqui F, Morselli AM, Gambineri A, Pasquali R. Dietary habits and their relationship with hormones and metabolism in overweight and obese women with polycystic ovary syndrome. Clin Endocrinol 2013; 78: 52-9.

79. Chavarro JE, Rich-Edwards JW, Rosner BA, Willett WC. A prospective study of dietary carbohydrate quantity and quality in relation to risk of ovulatory infertility. Eur J Clin Nutr. 2009;63:78-86.

80. Mutsaerts MA, van Oers AM, Groen H, Burggraaff JM, Kuchenbecker WK, Perquin DA, Koks CA, van Golde R, Kaaijk EM, Schierbeek JM, et al. Randomized trial of a lifestyle program in obese infertile women. N Engl J Med 2016; 374: 1942-53.

81. Kulak D, Polotsky AJ. Should the ketogenic diet be considered for enhancing fertility? Maturitas 2013; 74 10-3.

82. McGrice M, Porter J. The effect of low carbohydrate diets on fertility hormones and outcomes in overweight and obese women: A systematic review. Nutrients 2017; 27; 9. pii: E204.

83. Tay J, Luscombe-Marsh ND, Thompson CH, Noakes M, Buckley JD, Wittert GA, et al. Comparison of low- and high-carbohydrate diets for type 2 diabetes management: a randomized trial. Am J Clin Nutr 2015; 102 (4): 780-90. 